\title{
Implementing emergency department-based HIV testing in a low-resource setting: The value of a structured feasibility assessment tool
}

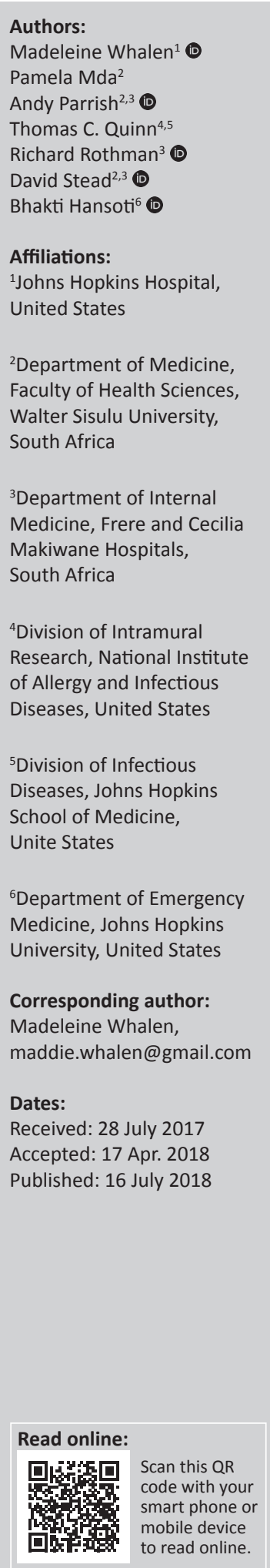

Introduction: $\mathrm{HIV}$ is a worldwide health problem with continuing high rates of new infections in many parts of the world. This lack of progress in decreasing overall incidence rates has sparked innovative HIV testing strategies, including expansion of testing into the emergency department (ED) setting. Emergency departments have been shown to be high-yield testing venues in the United States and other developed world settings. The feasibility of expanding public health HIV services in the ED in limited-resource countries is unclear.

Methods: We performed a cross-sectional feasibility assessment of a convenience sample of four hospitals in the Eastern Cape, South Africa. We administered three adapted interview tools from a previously field-tested survey instrument at each facility (total of 10 interviews) to gather an overview of the health facility, their HIV counselling and testing services, and their laboratory services.

Results: All of the health facilities had access to basic commodities such as water and electricity. Many had severe human resource limitations and provided care to wide population catchment areas. In addition, there was little integration of HIV testing into current daily ED operations. Hospital staff identified numerous barriers to future ED testing efforts.

Conclusions: Although control of the HIV epidemic requires innovative testing strategies and treatment, specific assessments are warranted on how to incorporate routine HIV testing into an acute care facility like the ED, which typically has many competing priorities. The use of a prospective structured tool incorporating both barriers and benefits can provide valuable field-tested guidance for increased programme planning for HIV testing.

\section{Introduction}

The Joint United Nations Programme on HIV/AIDS (UNAIDS) estimates that more than 10 million people worldwide have contracted HIV since $2011 .{ }^{1}$ Despite public health efforts in key areas, such as West and Central Africa, declines in new HIV infection rates are 'marginal'. ${ }^{2}$ To address stagnated HIV incidence, the World Health Organization (WHO), the United Kingdom's Health Protection Agency and the United States' Centers for Disease Control and Prevention (CDC) recommend expanded and integrated HIV testing strategies. This includes testing for all healthcare clients, including those utilising emergency services. ${ }^{3,45}$ The inclusion of the emergency department (ED) represents a viable and effective strategy for HIV testing in both the highresource and resource-limited settings. Emergency departments provide unrestricted access to large numbers of patients who may not otherwise interact with the healthcare system, and have been shown to effectively identify new HIV infections in high-resource settings. ${ }^{6,7,8}$ Studies that have sought to quantify the effectiveness of ED-based HIV testing in low-resource settings are limited but have found an HIV prevalence of $2 \%-43 \%$ with a proportion of $65 \%-90 \%$ of previously undiagnosed HIV infection. ${ }^{9}$ In addition, EDs care for key high HIV-risk populations, such as drug and alcohol users, young men and sex workers who are missed in more traditional settings such as antenatal clinics. ${ }^{1,10}$

Despite the global HIV burden, national and international recommendations, as well as the demonstrated efficacy of ED-based testing in the United States, implementation has been inconsistent. A 2009 survey found that only 22\% of EDs provided routine HIV testing, and of

How to cite this article: Whalen M, Mda P, Parrish A, et al. Implementing emergency department-based HIV testing in a low-resource setting: The value of a structured feasibility assessment tool. S Afr J HIV Med. 2018;19(1), a793. https://doi.org/10.4102/sajhivmed. v19i1.793

Copyright: ( 2018. The Authors. Licensee: AOSIS. This work is licensed under the Creative Commons Attribution License. 
those, less than a third followed the recommended 'opt-out' format in the United States. ${ }^{11}$ A 2013 meta-analysis of HIV testing in the United Kingdom found that only $27.2 \%$ of eligible patients, as defined by the 2008 British HIV Association Guidelines, received HIV testing. ${ }^{12}$ Likewise, South African National Testing Guidelines call for universal HIV testing in all health facilities, including the ED, with pre- and post-test counselling; however, it is rarely implemented. ${ }^{13}$

Given this gap between practice and policy, a structured feasibility assessment may provide insight into barriers to implement effective and efficient ED-based HIV testing. Feasibility assessments are a key early component of dissemination and implementation of science and can have a large effect on an intervention's acceptability, which in turn influences adoption, penetration and sustainability. ${ }^{14}$ The use of an appropriate and efficient assessment tool addresses the implementation component of a theoretical recommendation and provides prospective data on human and physical resources that need to be developed or supplemented to truly incorporate HIV testing into sustainable standards of practice. In addition, the use of a tool goes above and beyond more common feasibility assessments which do not proactively gather data but rather implement screening and then retrospectively evaluate their success using testing uptake as a proxy for feasibility. ${ }^{15,16,17,18,19,20,21}$ Assessment tools are available from the WHO, Family Health International, Partners in Health and other HIV / AIDS organisations. ${ }^{22,23,24,25}$ In this study, we seek to evaluate the usability and importance of a preimplementation facility assessment tool in a low-resource setting where implementation of ED-based HIV testing is desperately needed.

\section{Methods}

We performed a cross-sectional feasibility assessment of a convenience sample of four tertiary hospitals in the Eastern Cape of South Africa. Hospitals were selected in the Eastern Cape based on geographic accessibility, existing relationships with participating institutions and the prioritisation of research efforts by the Medical Research Council of South Africa. Institutional Review Boards at Johns Hopkins University and Walter Sisulu University approved the study (IRB number IRB00105801).

\section{Survey instrument}

We utilised three interview tools in accordance with guidelines from Family Health International's Health Facility Tools to Assess Preparedness for HIV Service Delivery. ${ }^{23}$ The Guide consists of 13 tools that were designed to rapidly and comprehensively gather data regarding the availability and quality of essential elements of HIV services, organise and analyse the data, and plan for programme implementation. The Guide has been field tested in Cambodia, Ethiopia, Nigeria, Kenya, Senegal and Zambia and was designed to

\begin{tabular}{|ll|}
\hline Tool 1: Overview of the health facility & \\
\hline - Facility background & 7 questions \\
- Human resources & 10 questions \\
- Infrastructure and supplies & 17 questions \\
- Health services & 5 questions \\
- Guidelines and protocols & 5 questions \\
- Perspectives, maximising acceptability of ART & 7 questions \\
and ART start-up programmes & \\
- Interviewer observations & 14 questions \\
\hline Tool 4B: Counseling and testing services & 4 questions \\
\hline - General & \\
\hline - Thinking forward & \\
\hline Tool 6: Laboratory services and commodities & 5 questions \\
\hline
\end{tabular}

FIGURE 1: Tools summary.

be flexible for adaption to meet individual site needs. ${ }^{23}$ The interview tools in our study were selected by both international and local researchers (authors M.W., B.H. and P.M.) based on applicability to the South African ED setting. Two of the tools were administered without change from the original text. The third tool, an assessment of laboratory services, was modified to only include information that could not be gathered from review of the National Health Laboratory Service of South Africa Scope of Services. ${ }^{26}$ The interview tools included yes/ no, closed- and open-ended questions, as well as interviewer observations. This specific feasibility assessment toolkit was chosen based on a review of available tools and its previous use in low-resource settings. An overview of each of the three tools can be seen in Figure 1. The three tools in their entirety can be found at http:/ / www.aidsdatahub.org/sites/default/ files / documents / Health_Facility_Tools_to_Assess_ Preparedness_for_HIV_Services_Delivery_Including Antiretroviral_Therapy.pdf.

\section{Data collection}

Interviewees consented to using the provided script and delivered their responses orally or via email. In-person interviews were conducted in the interviewees' private offices. All interviews were conducted in English by the Johns Hopkins University researcher (M.W.) with facilitation from the Walter Sisulu University research partner (P.M.).

\section{Data analysis}

All answers were recorded on the paper survey tools and later entered into Excel for side-by-side analysis of each question for all four sites. The majority of questions were closed-ended and direct responses were recorded. For open-ended questions, the researcher listed each response verbatim, then combined and tallied repeated answers. Interviewees selected answers from a finite list regarding 
barriers and benefits to ED-based HIV testing as well as provided open-ended responses. These answers were then combined into one table. Narrative answers that commented on the same problem, but with slightly different language (e.g. 'manpower' and 'staff'), were grouped.

\section{Results}

A total of 10 interviews were completed across four sites. Information was gathered from four lab supervisors, three physicians and three nurses. Interviewees are identified in Table 1.

\section{Facility characteristics}

All facilities are state-run tertiary academic hospitals and operate under Walter Sisulu University. All sites have 24-hour access to electricity (with back-up generators), water and computers. None of the EDs have designated laboratory personnel to perform point-of-care testing, and only one site, LH, has an in-department, part-time pharmacist. In-depth facility descriptions are shown in Table 2 .

\section{Health services}

Each of the four hospitals has inpatient and outpatient departments. In addition, all of the EDs provide physicianinitiated, targeted HIV testing. Patients found to be HIVpositive are referred to their community antiretroviral (ARV) clinic using a standard referral form with the exception of Livingstone Hospital, which performs direct doctor-to-doctor sign-out on referred patents. Available health services at all hospitals include rapid and diagnostic HIV testing, ARV clinic, social services, pharmacy and postexposure prophylaxis for employees only. Frere Hospital,

TABLE 1: Interviewee role identification.

\begin{tabular}{lll}
\hline Facility & \multicolumn{2}{c}{ Interviewee } \\
\cline { 2 - 3 } & Too1 1 and Tool 4b & Tool 6 \\
\hline FH & Area manager (professional nurse) & Lab manager \\
NMH & $\begin{array}{l}\text { Head of Department (physician) and nurse in } \\
\text { charge (professional nurse) }\end{array}$ & Chemical pathologist \\
LH & $\begin{array}{l}\text { Head of Department (physician) and area manager } \\
\text { (professional nurse) }\end{array}$ & Lab manager \\
CMH & Head of Infectious Disease (physician) & Lab manager \\
\hline
\end{tabular}

LH, Livingstone Hospital; CMH, Cecilia Makiwane Hospital; FH, Frere Hospital; NMH, Nelson Mandela Hospital.

TABLE 2: Facility descriptions.

\begin{tabular}{|c|c|c|c|c|}
\hline Facility & Type & Location & ED patients per day & Human resource availability \\
\hline $\mathrm{FH}$ & Urban & East London & $140-150$ & $\begin{array}{l}\text { Medical doctors: } 11 \\
\text { Nursing staff: } 45 \\
\text { HIV tester and counsellors: } 0\end{array}$ \\
\hline $\mathrm{NMH}$ & Rural & Mthatha & $30-50$ & $\begin{array}{l}\text { Medical doctors: } 11 \\
\text { Nursing staff: } 24 \\
\text { HIV tester and counsellors: } 0\end{array}$ \\
\hline LH & Urban & Port Elizabeth & $260-330$ & $\begin{array}{l}\text { Medical doctors: } 14 \\
\text { Nursing staff: } 84 \\
\text { HIV tester and counsellors: } 0\end{array}$ \\
\hline $\mathrm{CMH}$ & Rural & Mdantsane & $80-100$ & $\begin{array}{l}\text { Medical doctors: } 10 \\
\text { Nursing staff: } 34 \\
\text { HIV tester and counsellors: } 0\end{array}$ \\
\hline
\end{tabular}

LH, Livingstone Hospital; CMH, Cecilia Makiwane Hospital; FH, Frere Hospital; NMH, Nelson Mandela Hospital.

$\dagger$, Number of full-time, dedicated staff.

$\$$, Includes professional nurses, staff nurses, enrolled nurses and nursing assistants.

ED, emergency department.
Nelson Mandela Hospital and LH have on-site National Health Laboratories and are able to perform all HIV-related testing. Cecilia Makiwane Hospital sends specimens offsite for testing.

\section{Benefits and barriers to emergency department-based HIV testing}

In addition to concrete information regarding provision of services related to HIV in the ED, we gathered subjective information from key informants regarding benefits and barriers to HIV testing in the ED. Responses to both open and closed-ended questions regarding testing barriers and potential benefits are included in Table 3. Each response is followed by the frequency it was listed in parentheses.

\section{Discussion}

The use of a structured feasibility assessment tool demonstrated concrete barriers to ED-based HIV testing that will need to be addressed at the institutional level prior to implementing an ED-based HIV testing strategy. However, some of the identified barriers are likely to spark controversy and will require strategies for improvement beyond the ED. Use of a structured tool informed understanding of staffing shortages (mix as well as amount), the structure of ED services in the context of the institution and cultural barriers. The descriptive nature of these interview tools brings new illustrative information to the study of HIV testing implementation. ${ }^{27}$

This assessment clearly demonstrated the lack of available human resources to take on new responsibilities and projects, such as implementation of ED-based HIV testing. The number of full-time ED nurses ranged from 24 to 84 to serve large daily volumes of patients. Emergency department physician staffing only ranged from 11 to 14 full-time providers to serve the same population. The shortage of nurses and doctors in South Africa, specifically those with emergency training, is an ongoing problem and will need to be factored into any new programme implementation. ${ }^{8,28,29}$ In addition, none of the facilities has ancillary staff that could potentially take on the new responsibility of HIV testing and counselling, such as social workers, laboratory technicians or other cadres. These numbers demonstrate a clear barrier to ED-based HIV testing and help to create a more robust picture of the human resource constraints to successful implementation of the HIV testing policy. The question of availability and willingness of staff is

TABLE 3: Opportunities and barriers to emergency department-based HIV testing.

\begin{tabular}{ll}
\hline Benefits & Barriers \\
\hline -Improved coordination with & -Staff shortages (4) \\
HIV referral system & -Physical space (2) \\
-Availability of reinforced & -Perception that the ED is not the place for \\
ARV adherence counselling & HIV testing as there are other dedicated HIV \\
-Sharing of knowledge with & testing resources (duplication of services) (3) \\
outpatient clinics & -Lack of time \\
& -Concerns regarding LTC (2) \\
& -Funding (2) \\
& -Insufficient staff education (2) \\
& - Health policy that HIV testing should be referred \\
& to clinics \\
& - Hospital policy does not allow for medication \\
& prescriptions for more than 7 days making it \\
& difficult to start ARVs \\
\hline
\end{tabular}

ARV, antiretroviral; ED, emergency department, LTC, linkage to care. 
cited in many accounts of ED-based HIV testing in both highand low-resource settings. ${ }^{20,30,31,32}$

The feasibility assessment tool provided a structured approach to garner information at each of the facilities and how the ED functions as a part of the whole. For all facilities, HIV testing has been systematically and purposefully shifted to outpatient and community ARV clinics. ${ }^{13}$ This system of assigning a task to the most basic facility that can fulfil it has created both cultural and structural barriers to ED-based HIV testing, as well as missed opportunities to test new patients. None of the four EDs provided universal HIV testing, and none currently has the resources to initiate ARVs. This is problematic in the current HIV environment that calls for non-targeted testing and treatment regardless of symptoms, viral load or CD4+ count. ${ }^{33}$ In addition, the processing time for laboratory tests may not be conducive to the fast pace of the ED environment.

Finally, the structured interviews recorded perceived benefits and barriers to HIV testing. This information reinforced and illuminated much of the information gathered through the objective interview tools. Gathering these data describes institutional knowledge and individual attitudes. Items such as health and hospital policy need to be addressed in order to successfully introduce a change in practice and culture. In addition, the perception that the ED is not the venue for testing for communicable diseases appears to be ingrained in hospital culture, and a campaign to challenge this assumption may need to be incorporated into project planning. The prevalent themes of lack of provider knowledge, costs, insufficient time, insufficient space and linkage to care (LTC) issues are apparent in this setting and echo concerns noted in ED-barrier studies completed in the United States and the United Kingdom. $27,31,32,34,35,36$ The overall concept of reframing the role of the ED in public health initiatives is a prevalent theme in both high- and low-resource settings. ${ }^{20,31,35}$ In addition, Mumma and Suffaletto ${ }^{30}$ highlight the need for public health officials to recognise the many competing priorities of the ED and create a collaborative project that meets both the acute care and public health needs of the population..$^{30}$

Implementation of Family Health International's interview guides was simple, and the language was accessible to the interviewees, including those for whom English was not a first language. Some of the questions required both high-level institutional knowledge and department-level statistics (e.g. number of full-time and part-time employed nurses) that was difficult for some respondents to provide. The questionnaires are also lengthy which makes sustained quality data collection difficult, given that it requires interviews with key informants in busy leadership positions. In addition, the key question of funding a new HIV testing initiative is not addressed in this assessment. Our study did not utilise Family Health International's provider or client attitudes tools but rather incorporated a more in-depth attitudes interview that included healthcare provider stigma and patient HIV knowledge at $\mathrm{FH}$ that has been previously described in this journal. ${ }^{37}$ Future pre-implementation efforts will need to continue to gather information on acceptability at all sites as it is a large component of cultural feasibility.
The use of this feasibility assessment provided essential information at the hospital level that, if expanded, could be used to influence policy at the institutional, provincial, state and even national level. In addition, given the documentation of the shortages of physical and human resources as well as staff buy-in found in this, and other feasibility assessments, it may be unethical to mandate new HIV testing strategies without accompanying solutions. ${ }^{31,35}$

In order to further inform the feasibility of ED-based HIV testing in these facilities, it will be important to administer both patient and provider questionnaires at all sites to evaluate cultural barriers, including stigma. ${ }^{37}$ In addition, the cost-effectiveness and financial feasibility of implementing a novel HIV testing programme will need to be studied. Given the time-prohibitive length of the surveys, it may also be beneficial to administer the tools in a setting that already provides ED-based HIV testing to determine which questions truly add value. This abbreviated questionnaire could then be validated. A priority matrix may also be a helpful prioritisation tool to organise results.

As this study was performed with a small convenience sample of four hospitals, it is limited in its generalisability to other institutions. The range of hospital resources and capacity is widely varied not only throughout South Africa but also across low-resource settings. In addition, interviewees provided information from their knowledge base and catchment areas; ED volumes, staffing numbers and laboratory times are estimates. Finally, bias is inherent to qualitative interviewing techniques. Structured interview guides were used to minimise interviewer level variation, but it cannot be completely eliminated. ${ }^{38}$

\section{Conclusion}

This feasibility assessment helped to inform the possibility of initiating ED-based HIV testing by establishing an anticipatory list of barriers that will need to be addressed prior to initiation of a new testing strategy. Although potentially high yield, ED-based HIV testing in South Africa will need to have dedicated resources to shift the cultural perception of acute care versus public health mandates, as well as physical limitations such as funding, staff and space. Possible solutions to these obstacles could be incorporation of more traditional strategies, such as clinic-based testing, diverting testing resources to the inpatient setting owing to the captive audience of patients, or provision of at-home or self-testing kits to all ED patients. ${ }^{39,40}$ Taking advantage of blood samples already sent to the laboratory for add-on testing may also increase HIV testing volumes while minimising performing point-of-care testing on frontline staff. ${ }^{41,42}$

\section{Acknowledgements}

This research was made possible by the staff and CEOs of Frere Hospital, Cecilia Makiwane Hospital, Livingstone Hospital, Nelson Mandela Hospital, Walter Sisulu University, 
and supported in part by the Division of Intramural Research, NIAID, NIH and Johns Hopkins Department of Emergency Nursing.

\section{Competing interests}

The authors declare no potential conflict of interest.

\section{Authors' contributions}

M.W. designed and conducted the study, analysed the data and drafted the manuscript. P.M. contributed to the study design and provided revisions to the manuscript. A.P., D.S., R.R. and T.C.Q. contributed to the drafting and revision of the manuscript. B.H. oversaw the study and contributed to the study design, data analysis and drafting and editing of the manuscript.

\section{References}

1. UNAIDS. Prevention gap report [homepage on the Internet]. 2016 [cited 2017 Mar 28]. Available from: http://www.unaids.org/en/resources/documents/2016/ prevention-gap

2. 90-90-90: An ambitious treatment target to help end the AIDS epidemic. Geneva: UNAIDS; 2014.

3. World Health Organization. Consolidated guidelines on HIV testing services. Geneva: World Health Organization; 2015.

4. British HIV Association (BHIVA), British Association for Sexual Health and HIV (BASHH), British Infection Society (BIS). UK national guidelines for HIV testing. London: The British HIV Association; 2008.

5. Branson BM, Handsfield HH, Lampe MA, et al. Revised recommendations for HIV testing of adults, adolescents, and pregnant women in health-care settings. MMWR Recomm Rep. 2006;55(RR-14):4.

6. Hsieh Y, Kelen GD, Laeyendecker O, Kraus CK, Quinn TC, Rothman RE. HIV care continuum for HIV-infected emergency department patients in an inner-city academic emergency department. Ann Emerg Med. 2015;66(1):69-78. https:// doi.org/10.1016/j.annemergmed.2015.01.001

7. Menchine $M$, Zhou M, Lotfipour S, Chakravarthy B. Moving beyond screening: How emergency departments can help extinguish the HIV/AIDS epidemic. West J Emerg Med. 2016;17(2):135. https://doi.org/10.5811/westjem.2016.1.29100

8. Brysiewicz P, Bruce J. Emergency nursing in South Africa. Int Emerg Nurs. 2008;16(2):127-131. https://doi.org/10.1016/j.ienj.2008.01.001

9. Hansoti B, Kelen GD, Quinn TC, et al. A systematic review of emergency department based HIV testing and linkage to care initiatives in low resource settings. PLoS One. 2017;12(11):e0187443. https://doi.org/10.1371/journal.pone.0187443

10. Shisana O, Rehle T, Simbayi LC, et al. South African national HIV prevalence, incidence and behaviour survey, 2012. Cape Town: HSRC Press; 2014.

11. Rothman RE, Hsieh Y, Harvey L, et al. 2009 US emergency department HIV testing practices. Ann Emerg Med. 2011;58(1):S9. e4.

12. Elmahdi R, Gerver SM, Gomez Guillen G, Fidler S, Cooke G, Ward H. Low levels of HIV test coverage in clinical settings in the U.K.: A systematic review of adherence to 2008 guidelines. Sex Transm Infect. 2014;90(2):119-124. https://doi.org/10.1136/ sextrans-2013-051312

13. National Department of Health. National HIV counselling and testing policy guidelines. Health Department: Republic of South Africa; 2015.

14. Brownson RC, Colditz GA, Proctor EK. Dissemination and implementation research in health: Translating science to practice. Oxford University Press; 2012.

15. Burns F, Edwards SG, Woods J, et al. Acceptability, feasibility and costs of universal offer of rapid point of care testing for HIV in an acute admissions unit: Results of the RAPID project. HIV Med. 2013;14(S3):10-14. https://doi.org/10.1111/hiv.12056

16. Ellis S, Graham L, Price DA, Ong EL. Offering HIV testing in an acute medical admissions unit in Newcastle upon Tyne. Clin Med (Lond). 2011;11(6):541-543. https://doi.org/10.7861/clinmedicine.11-6-541

17. Hempling MC, Zielicka-Hardy A, Ellis JP, Majewska W, Fida G. Routine HIV testing in the emergency department: Feasible and acceptable? Int J STD AIDS 2016;27(14):1267-1274. https://doi.org/10.1177/0956462415613727

18. Rayment M, Thornton A, Mandalia S, et al. HIV testing in non-traditional settingsthe HINTS study: A multi-centre observational study of feasibility and acceptability. PLoS One. 2012;7(6):e39530. https://doi.org/10.1371/journal.pone.0039530
19. Rayment M, Rae C, Ghooloo F, et al. Routine HIV testing in the emergency department: Tough lessons in sustainability. HIV Med. 2013;14(S3):6-9. https:// doi.org/10.1111/hiv.12069

20. Waxman MJ, Kimaiyo S, Ongaro N, Wools-Kaloustian KK, Flanigan TP, Carter EJ. Initial outcomes of an emergency department rapid HIV testing program in western Kenya. AIDS Patient Care STDS 2007;21(12):981-986. https://doi.org/10.1089/ apc.2007.0075

21. Nakanjako D, Kamya M, Daniel K, et al. Acceptance of routine testing for HIV among adult patients at the medical emergency unit at a national referral hospital in Kampala, Uganda. AIDS Behav. 2007:11(5):753-758. https://doi.org/10.1007/ s10461-006-9180-9

22. World Health Organization. Service availability and readiness assessment (SARA): An annual monitoring system for service delivery: Reference manual. 2013.

23. Harkins J. Health facility tools to assess preparedness for HIV services delivery including antiretroviral therapy. Arlington, VA: Family Health International, 2007; p. 242.

24. Leung $C$, Aris E, Mhalu A, et al. Preparedness of HIV care and treatment clinics for the management of concomitant non-communicable diseases: A cross-sectiona survey. BMC Public Health. 2016;16(1):1002. https://doi.org/10.1186/s12889016-3661-1

25. Health Research and Educational Trust. HIV testing in emergency departments: A practical guide [homepage on the Internet]. 2009 [cited 2017 Mar 28]. Available from: http://www.edhivtestguide.org/EDHIPrin-4319.htm

26. National Health Laboratory Service. Scope of services [homepage on the Internet] 2017 [cited 2017 Mar 28]. Available from: http://www.nhls.ac.za/?page=scope of_services\&id=26

27. Arbelaez $\mathrm{C}$, Wright $\mathrm{EA}$, Losina $\mathrm{E}$, et al. Emergency provider attitudes and barriers to universal HIV testing in the emergency department. J Emerg Med. 2012;42(1):7-14. https://doi.org/10.1016/j.jemermed.2009.07.038

28. Wallis LA, Garach SR, Kropman A. State of emergency medicine in South Africa. Int J Emerg Med. 2008;1(2):69-71. https://doi.org/10.1007/s12245-008-0033-3

29. Coovadia $H$, Jewkes R, Barron $P$, Sanders D, Mclntyre D. The health and health system of South Africa: Historical roots of current public health challenges. Lancet. 2009;374(9692):817-834. https://doi.org/10.1016/S0140-6736(09)60951-X

30. Mumma BE, Suffoletto BP. Less encouraging lessons from the front lines: Barriers to implementation of an emergency department-based HIV screening program. Ann Emerg Med. 2011;58(1):S48. https://doi.org/10.1016/j.annemergmed.2011. 03.022

31. Burke RC, Sepkowitz KA, Bernstein KT, et al. Why don't physicians test for HIV? A review of the US literature. AIDS. 2007:21(12):1617-1624. https://doi.org/10.1097/ QAD.0b013e32823f91ff

32. Hecht CR, Smith MD, Radonich K, Kozlovskaya O, Totten VY. A comparison of patient and staff attitudes about emergency department-based HIV testing in 2 urban hospitals. Ann Emerg Med. 2011;58(1):S32.e4.

33. World Health Organization. Progress report 2016: Prevent HIV, test and treat all: WHO support for country impact. Geneva: World Health Organization; 2016.

34. Irvin CB, Flagel BT, Fox JM. The emergency department is not the ideal place for routine HIV testing. Ann Emerg Med. 2007;49(5):722. https://doi.org/10.1016/j. annemergmed.2007.03.011

35. Waxman MJ, Popick RS, Merchant RC, Rothman RE, Shahan JB, Almond G. Ethical, financial, and legal considerations to implementing emergency department HIV screening: A report from the 2007 conference of the National Emergency Department HIV Testing Consortium. Ann Emerg Med 2011;58(1):S43. https:// doi.org/10.1016/j.annemergmed.2011.03.021

36. Hsieh Y, Jung JJ, Shahan JB, Moring-Parris D, Kelen GD, Rothman RE. Emergency medicine resident attitudes and perceptions of HIV testing before and after focused training program and testing implementation. Acad Emerg Med. 2009;16(11):1165-1173. https://doi.org/10.1111/j.1553-2712.2009.00507.x

37. Hansoti B, Hill SE, Whalen $M$, et al. Patient and provider attitudes to emergency department-based HIV counselling and testing in South Africa. S Afr J HIV Med. 2017;18(1):1-7.

38. Broom A. Using qualitative interviews in CAM research: A guide to study design data collection and data analysis. Complement Ther Med. 2005;13(1):65-73. https://doi.org/10.1016/j.ctim.2005.01.001

39. Liddicoat RV, Horton NJ, Urban R, Maier E, Christiansen D, Samet JH. Assessing missed opportunities for HIV testing in medical settings. J Gen Intern Med. 2004;19(4):349-356. https://doi.org/10.1111/j.1525-1497.2004.21251.x

40. Gaydos CA, Hsieh Y, Harvey L, et al. Will patients 'opt in' to perform their own rapid HIV test in the emergency department? Ann Emerg Med. 2011;58(1):S78. https://doi.org/10.1016/j.annemergmed.2011.03.029

41. Lyss SB, Branson BM, Kroc KA, Couture EF, Newman DR, Weinstein RA. Detecting unsuspected HIV infection with a rapid whole-blood HIV test in an urban emergency department. J Acquir Immune Defic Syndr. 2007;44(4):435-442. https://doi.org/ 10.1097/QAl.0b013e31802f83d0

42. Cornett JK, Kirn TJ. Laboratory diagnosis of HIV in adults: A review of current methods. Clinical Infect Dis. 2013;57(5):712-718. https://doi.org/10.1093/cid/ cit281 